\title{
On Variables Sampling Plans for Food Safety
}

\author{
Edgar Santos-Fernández (I), K Govindaraju (I), Geoff Jones (I)
}

(I) MU - Institute of Fundamental Sciences, Massey University (Tennent Dr, Palmerston North 4474. New Zealand)

\section{Resumo}

Introduction: Acceptance sampling methodology is used for disposition of lots of commodities as suitable to be consumed. It provides assurance to the consumers on the quality and safety of the accepted batches. Variables inspection plans are advantageous since it requires smaller sample sizes in relation to plans for attributes. This research studied the sampling performance when the analytical tests where done using composite samples. A novel sampling plan for variables is presented. It discusses the sampling performance when the pathogens are heterogeneously distributed among the batches. Materials and Methods: The inspection of bulk materials commonly involves composite sampling which allows sampling economy and a reduction of the laboratory effort. We discuss the effect of perfect and imperfect composite sample preparation on the performance of two and three-class sampling inspection plans by variables. A common assumption in variables plans is that the frequencies of microorganism are lognormally distributed and the traditional proceeding for variables is used after applying logarithm base 10. A new approach based on the angular (sinh-arcsinh) transformation is proposed. The performance of the different sampling alternatives was evaluated using Monte Carlo simulation. Results and Conclusion: The results show the sampling economy when compositing under the assumption of the perfect mixing of primary samples or increments. However, in the processes characterized if there was an inefficient mixing, the effect of dilution will not be compensated by improving the sampling performance, therefore the consumer's risks will not be reduced by compositing. Results show at least eight sample increments should be used, particularly if the sublot variability differs 
hugely. On the other hand, the suggested transformation produces a more stringent plan in comparison with the classical variables plan. This method lowers the consumer's risk at the same risk for the producer and shows a higher robustness when the true statistical distribution is other than lognormal and in the presence of contamination.

Palavras-Chave: Sampling plan, Composite sampling, Mixing, sinh-arcsinh transformation

Agência de Fomento: 